\title{
Do slow and fast gamma rhythms correspond to distinct functional states in the hippocampal network?
}

\author{
Laura Lee Colgin \\ Center for Learning and Memory \\ Department of Neuroscience \\ The University of Texas at Austin \\ 1 University Station Stop C7000 \\ Austin, TX 78712 \\ USA \\ Phone +1 (512) 232-6361 \\ Fax: $+1(512) 475-8000$ \\ Email: colgin@mail.clm.utexas.edu
}




\begin{abstract}
For decades, hippocampal gamma was thought to be a single type of rhythm with a continuously varying frequency. However, an increasing body of evidence supports a new hypothesis regarding hippocampal gamma. The patterns traditionally defined as hippocampal gamma may actually comprise separate gamma subtypes with distinct frequencies and unique functions. The present review discusses the evidence for and against this new viewpoint. This review will also point out key questions that remain to be answered to validate the two-gamma hypothesis.
\end{abstract}

Keywords: Gamma; hippocampus; rhythms; oscillations; entorhinal cortex 


\section{Background}

Until fairly recently, gamma rhythms in the hippocampus of freely behaving rodents received little experimental attention. There are several likely explanations as to why many hippocampal researchers may have disregarded gamma. One reason may be that gamma rhythms are overshadowed by larger amplitude patterns in hippocampal local field potentials, namely theta rhythms (Colgin, 2013, for a review) and sharp waves (Buzsaki, 1986). While theta and sharp waves have clear behavioral correlates, including active exploration for theta and immobility for sharp waves, gamma rhythms are observed during all behavioral states (Buzsaki et al., 1983). It is difficult to develop a testable hypothesis to explain a rhythm's function if the rhythm's occurrence is not limited to specific behaviors. Moreover, gamma rhythms are exceptionally variable with regard to both their amplitude and their frequency (from $\sim 25 \mathrm{~Hz}$ to $\sim 100 \mathrm{~Hz}$ ). Effects of experimental manipulations on gamma amplitude and frequency can be difficult to detect because baseline measures of gamma amplitude and frequency continuously vary. Some sources of hippocampal gamma amplitude, or power, variations were explained in the relatively early days of hippocampal gamma research. Gamma power is maximal during theta-related behaviors, and the power of theta-associated gamma regularly fluctuates as a function of theta phase (Buzsaki et al., 1983; Bragin et al., 1995). These findings regarding gamma amplitude did not address, however, the occurrence of gamma during non-theta states (e.g., immobility). These findings also did not address variations in gamma frequency.

\section{One gamma rhythm with continuously varying frequency?}

In an initial important study from the Buzsaki Lab, relatively fast gamma $(\sim 100 \mathrm{~Hz})$ was associated with relatively fast theta $(\sim 10 \mathrm{~Hz})$, and slower gamma $(\sim 50 \mathrm{~Hz})$ was associated with slower theta $(\sim 6 \mathrm{~Hz})$ (Bragin et al., 1995). This finding could indicate that gamma-generating circuitry speeds up as running speed increases, considering that theta frequency increases with

running speed (Rivas et al., 1996; Slawinska and Kasicki, 1998; Jeewajee et al., 2008). This conclusion is consistent with recent findings showing that gamma frequency increases with running speed (Ahmed and Mehta, 2012). Increases in gamma frequency with increased running speed were accompanied by increases in the firing rate of interneurons. This corresponding interneuron effect may underlie gamma frequency increases considering that gamma rhythms involve inhibitory postsynaptic events (Soltesz and Deschenes, 1993; Penttonen et al., 1998; Pernia-Andrade and Jonas, 2014). These findings suggest that speed-modulated interneurons drive increases in gamma frequency as running speed increases to support faster transitions across gamma-modulated cell assemblies (Ahmed and Mehta, 2012).

However, some of the results shown in the Ahmed and Mehta (2012) work appear to show a split between lower $(\sim 30-40 \mathrm{~Hz})$ and higher $(>60 \mathrm{~Hz})$ gamma frequencies (see their Figure 3). This supports the hypothesis that separate low frequency ('slow') and high frequency ('fast') gamma rhythms exist and that these different gamma subtypes are differentially affected by running speed. In line with this idea, the distribution of power in the gamma frequency range 
(i.e., $25-100 \mathrm{~Hz}$ ) across different running speeds is best fit by two functions rather than a single function (Zheng et al., 2014). One function fits data in the slow gamma range, with slow gamma frequencies changing minimally as running speed increases. The other function fits the distribution of power in the fast gamma frequency range. In this case, frequencies increase as running speeds increase, as previously reported. These findings support the conclusion that slow and fast gamma states are more likely to occur during behaviors associated with low and high running speeds, respectively. Consistent with this conclusion, Kemere and colleagues also showed that slow gamma $(\sim 20-55 \mathrm{~Hz})$ power in CA1 decreased with running speed, while fast gamma $(\sim 65-140 \mathrm{~Hz})$ power increased with running speed (Kemere et al., 2013).

\section{Two gamma rhythms entrained by different hippocampal inputs?}

An early important study conducted by Bragin and colleagues (1995) employed current source density (CSD) analyses and concluded that there are two independent sources of hippocampal gamma: one in the entorhinal cortex (EC) and another intrinsic to the hippocampus. The dominant current sinks during gamma activity were observed in the middle molecular layer of the dentate gyrus, the termination zone for medial perforant path fibers. Moreover, CSD maps resembled CSD profiles for excitatory postsynaptic responses to medial perforant path stimulation. These findings suggest that the dominant generator of hippocampal gamma is the EC. Support for an entorhinal generator of hippocampal gamma was also provided by a CSD study in guinea pigs (Charpak et al., 1995). In the Bragin et al. study (1995), EC lesions were also performed. After the lesions, the dominant current sinks in the molecular layer disappeared, and gamma currents arose in CA1 stratum radiatum, the termination zone for Schaffer collateral axons from CA3. This finding suggested that CA3 was a second site of hippocampal gamma generation. Interestingly, the average frequency of hippocampal gamma decreased after the entorhinal lesions, a finding that could have occurred as a result of diminished excitatory drive to the network. A later study from the same lab provided additional evidence for two hippocampal gamma oscillators, one in the dentate gyrus and one in the CA3CA1 network. However, the peak frequency of these two gamma oscillators was reported to be similar, $\sim 53 \mathrm{~Hz}$ (Csicsvari et al., 2003).

More recent findings shed light on the significance of the EC lesion effects reported by Bragin et al. (1995). Colgin et al. (2009) employed paired recordings from CA1 and two of its inputs and reported separate slow and fast gamma subtypes that were linked to inputs from the medial entorhinal cortex (MEC) and CA3, respectively. Slow $(\sim 25-50 \mathrm{~Hz})$ gamma rhythms in CA1 were coupled with slow gamma in CA3, and fast gamma $(\sim 65-140 \mathrm{~Hz})$ rhythms in CA1 were coherent with fast gamma in MEC (see also Kemere et al., 2013, their Figure 3b, for a replication). Activity of $\mathrm{CA} 3$ neurons was significantly more correlated with the phase of slow gamma activity in CA1 than the phase of CA1 fast gamma. Also, layer III MEC neurons preferentially fired at a particular phase of fast, not slow, gamma rhythms in CA1. These findings support the hypothesis that distinct frequencies of gamma rhythms in the hippocampus are driven by different hippocampal inputs, namely CA3 and the EC. 
Consistent with this hypothesis, a recent report (Schomburg et al., 2014) detected separate theta-modulated gamma components in stratum radiatum (SR) and stratum lacunosummoleculare (S-LM) of CA1. The gamma activity in SR and S-LM displayed peak frequencies of $\sim 40 \mathrm{~Hz}$ and $\sim 90 \mathrm{~Hz}$, respectively (see also Belluscio et al., 2012). Moreover, another recent study provided support for the earlier entorhinal lesion results from Bragin et al. (1995): fast gamma $(\sim 80 \mathrm{~Hz})$ power in CA1 was significantly lower in mice in which the direct projection from MEC layer III to CA1 was blocked optogenetically (Yamamoto et al., 2014). Taken together, all of these findings support the conclusion that the EC drives a fast $(\sim 60-100 \mathrm{~Hz})$ variant of gamma in the hippocampus, while CA3 drives a slow $(\sim 30-50 \mathrm{~Hz})$ gamma subtype.

\section{Two gamma rhythms with separate functions?}

But, does the existence of distinct gamma subtypes with different frequencies have implications for mnemonic operations in the hippocampal network? An increasing number of studies suggest that slow and fast gamma carry out specific functions. Yet, no consensus has been reached as to what those functions are.

Several studies have suggested that slow gamma rhythms play an important mnemonic role in the hippocampal network. Place cell ensembles retrieve representations of upcoming locations when theta-modulated slow gamma $(25-55 \mathrm{~Hz})$ rhythms occur (Bieri et al., 2014). Additionally, Tort et al. (2009) found that coupling between theta phase and slow gamma (30-60 $\mathrm{Hz}$ ) amplitude in CA3 increased as animals learned to correctly choose context-dependent stimuli in order to receive a reward. In this study, enhancement of theta-slow gamma coupling correlated with task performance and occurred prior to the point when animals made the correct selection, implying that theta-modulated slow gamma facilitated retrieval of the contextdependent memory. Shirvalkar and colleagues (2010) subsequently reported that co-modulation of theta and slow gamma $(30-50 \mathrm{~Hz})$ power was significantly higher in trials when animals successfully remembered the correct choice in a match-to-place task compared to trials when errors were made. A later study found that slow gamma $(30-45 \mathrm{~Hz})$ power increased toward the end of a fixation period in a delayed spatial alternation task, a point when memory retrieval may occur (Takahashi et al., 2014). A recent study showed that $\sim 20-40 \mathrm{~Hz}$ coherence between lateral entorhinal cortex (LEC) and CA1 developed with learning of an odor-place association (Igarashi et al., 2014). The coherence occurred during odor sampling, as the odor presumably triggered recall of the associated place. Also, the development of $\sim 20-40 \mathrm{~Hz}$ coherence between LEC and CA1 was paralleled by the development of odor-specific responses in LEC and CA1 cells. Together, these findings support the conclusion that slow gamma promotes the retrieval of stored memories.

However, other results do not support this hypothesis of slow gamma function. Trimper and colleagues (2013) recently reported increases in slow gamma $(\sim 40 \mathrm{~Hz})$ coherence between CA3 and CA1 as rats explored novel objects, particularly objects that were later wellremembered. Memory encoding, not memory retrieval, would be expected to occur during 
exploration of novel objects. In their Discussion, the authors suggested that the observed slow gamma coherence could reflect retrieval of memories of other objects that had previously been presented in the same locations. This interpretation remains to be tested, though. Similarly, Kemere et al. (2013) found that slow gamma $(\sim 20-55 \mathrm{~Hz})$ power increased when animals explored a novel W-maze. However, fast gamma power also increased during exploration of the novel W-maze. Also, animals had been trained on a familiar $\mathrm{W}$-maze previously. Thus, it is possible that animals retrieved their memory of the general context of the W-maze task as they explored the new maze, considering that they had to perform the task correctly in order to receive a reward. Whatever the case may be, it is clear that more experiments are needed to understand how these various slow gamma results fit together.

What is the current leading hypothesis of fast gamma function? One hypothesis is that fast gamma facilitates encoding of current spatial information. Fast gamma coordinates activity across MEC and CA1 (Colgin et al., 2009; Kemere et al., 2013), and MEC transmits information to the hippocampus about current spatial location (Brun et al., 2002; Fyhn et al., 2004; Hafting et al., 2005; Brun et al., 2008). The hippocampus would require this current location information during encoding of spatial memories. Some results provide support for this memory encoding during fast gamma hypothesis. Scopolamine, a drug that impairs memory encoding but not memory retrieval, reduced fast $(\sim 60-120 \mathrm{~Hz})$ but not slow $(\sim 20-40 \mathrm{~Hz})$ gamma rhythms (Newman et al., 2013). The ratio of fast gamma $(\sim 55-95 \mathrm{~Hz})$ to slow gamma $(\sim 23-40 \mathrm{~Hz})$ power in CA1 was significantly higher when mice used current place cues to navigate rather than relying on their memory of spatial sequences (Cabral et al., 2014). Ensembles of place cells preferentially represented recent locations, rather than predicting upcoming locations, during periods of fast gamma $(\sim 60-100 \mathrm{~Hz})$ rhythms (Bieri et al., 2014). CA1 fast gamma $(60-90 \mathrm{~Hz})$ power was high at the start of a fixation period in a delayed spatial alternation task that included a light stimulus during the fixation period (Takahashi et al., 2014). The authors suggested that fast gamma likely occurred as the animal attended to the visual stimulus.

However, other studies yielded results that are not easily reconciled with a memory encoding function for fast gamma. In a study by Yamamoto et al. (2014), CA1 fast gamma $(\sim 65-100 \mathrm{~Hz})$ power was significantly higher on the central arm of a delayed spatial alternation task than on the side arms. Moreover, performance on this task was significantly impaired when CA1 fast gamma was suppressed on the central arm by inhibiting MEC layer III inputs to CA1. The central arm portion of this type of task is not thought to be associated with memory encoding but rather with memory retrieval (Montgomery and Buzsaki, 2007) or working memory (Yamamoto et al., 2014). In the latter case, memory retrieval may occur in the start box before the animal enters the central arm (Ainge et al., 2007). Yamamoto and colleagues (2014) did not test the effects of inhibiting MEC layer III inputs to CA1, and thereby inhibiting fast gamma, at the reward sites. Thus, it is possible that task impairment would also occur if fast gamma was blocked at the reward sites, where memory encoding likely occurs. Such results would be consistent with a memory encoding role for fast gamma. In any event, the results from 
Yamamoto et al. (2014) suggest that fast gamma may play a role in working memory. The afore-mentioned findings from Takahashi et al. (2014) could also be re-interpreted to support this hypothesis. Specifically, fast gamma power could have been high during the initial part of the fixation period because the animal was maintaining a memory of the previous trial in working memory. The working memory function hypothesis for fast gamma could also explain recent results from Schomburg et al. (2014). They reported increases in both slow gamma $(\sim 40 \mathrm{~Hz})$ and fast gamma $(\sim 90 \mathrm{~Hz})$ on the center arm of a delayed spatial alternation task. It is possible that slow gamma increased as animals retrieved their memory of the previous trial at the initial part of the center arm trajectory. Fast gamma may then have occurred during the remainder of the central arm trajectory as animals held this information in working memory.

In summary, the current hypotheses regarding fast gamma and slow gamma functions in memory processing require further testing. The hypotheses that fast gamma promotes memory encoding and slow gamma facilitates memory retrieval remain attractive in part because they are consistent with findings related to theta rhythms. Memory encoding and retrieval are thought to optimally occur at different phases of theta (Hasselmo et al., 2002; Kunec et al., 2005; Manns et al., 2007; Lever et al., 2010; Douchamps et al., 2013; Siegle and Wilson, 2014). Perhaps related to this, slow and fast gamma rhythms tend to occur on different theta phases (Colgin et al., 2009; Belluscio et al., 2012; Lasztoczi and Klausberger, 2014; Yamamoto et al., 2014; Schomburg et al., 2014), although there are discrepancies across studies with regard to what those phases are. The phase differences may be due to different methods, as suggested by Schomburg et al. (2014). Also, the preferred phase of fast gamma reported in Colgin et al. (2009) was likely distorted somewhat by epsilon ( 100-150 Hz) activity, which largely reflects spiking (Belluscio et al., 2012), because the fast gamma band in Colgin et al. extended up to $140 \mathrm{~Hz}$. Another possibility is that discrepancies across studies are related to differences in behavioral tasks, considering that coupling of gamma amplitude to theta phase has been related to task performance in several studies (see Colgin, 2014, for a review).

\section{Link between gamma rhythms and synaptic plasticity?}

'Theta burst stimulation' patterns (i.e., bursts of $100 \mathrm{~Hz}$ stimulation delivered at theta frequency) resemble theta-modulated fast gamma rhythms and are optimal for inducing longlasting increases in synaptic strength that are believed to underlie memory encoding (i.e., longterm potentiation (LTP); Larson et al., 1986; Larson and Lynch, 1986). This resemblance naturally leads to the hypothesis that theta-modulated fast gamma rhythms are involved in memory encoding, a hypothesis that has been suggested previously for theta-modulated gamma in general (Jensen and Lisman, 2005). In line with this hypothesis, the fast gamma period ( $10-$ $15 \mathrm{~ms}$ ) falls within the critical time window for inducing increases in synaptic strength (i.e., $\leq \sim 20 \mathrm{~ms}$; Bi and Poo, 1998). Also, there are some indications that cells in superficial layers of entorhinal cortex fire repetitively at fast gamma frequencies (Chrobak and Buzsaki, 1998) across successive theta cycles (e.g., Climer et al., 2014). Thus, it is plausible to hypothesize that entorhinal inputs to hippocampus during exploratory behaviors are capable of inducing LTP. 
There is also evidence suggesting that slow gamma $(40 \mathrm{~Hz})$ stimulation induces LTP when paired with postsynaptic depolarization (Buonomano, 1999). However, slow gamma stimulation in this study was delivered continuously, not timed by theta periods. There is little in vivo evidence that hippocampal principal cells fire at gamma frequencies for extended periods of time (i.e., >200 ms, as in the Buonomano, 1999 study). Identifying LTP induction patterns that are physiologically realistic is important, in part because not all forms of LTP are identical. For example, signaling pathways involved in LTP differ for natural (i.e., theta burst stimulation) and unnatural (i.e., tetanic stimulation) stimulation protocols (Zhu et al., in press). Nevertheless, the question of how slow gamma activation patterns relate to synaptic plasticity remains open and warrants further study.

\section{Slow and fast gamma during quiescent states and sleep}

Any explanation of the functional significance of slow and fast gamma must also address the occurrence of slow and fast gamma during quiescent and sleep states. Previous findings suggesting that slow and fast gamma coordinate the flow of activity in the hippocampal network (Colgin et al., 2009) evoke hypotheses regarding slow and fast gamma during different states of sleep and waking. Sharp waves involve propagation of activity from CA3 to CA1 (Buzsaki 1986; Colgin et al., 2004), suggesting the hypothesis that slow gamma occurs during sharp waverelated states, including awake immobility and slow-wave sleep (Buzsaki 1986). Theta rhythms occur during REM (Buzsaki et al., 1983), and activity flows from EC superficial layers to the hippocampus during theta (Chrobak and Buzsaki, 1994). Thus, a plausible hypothesis is that fast gamma occurs in the hippocampus during REM sleep. In line with the first hypothesis, recent studies revealed that slow gamma power increased in CA3 and CA1 during sharp wave-ripples (Carr et al., 2012) and decreased during REM (Schomburg et al., 2014). Fast gamma (60-120 $\mathrm{Hz}$ ) power, on the other hand, has been reported to increase in CA1 and MEC during REM sleep (Schomburg et al., 2014), consistent with our unpublished observations (Figure 1). These findings are in line with the hypothesis that slow gamma promotes CA3 inputs to CA1 and fast gamma facilitates EC inputs to CA1 during both sleep and waking states.

\section{Key questions for future study}

If slow and fast gamma are separate network states in the hippocampus, as the arguments above suggest, then one would expect the two states to have separate mechanisms of generation. A likely possibility is that different classes of interneurons are involved in slow and fast gamma generation. Consistent with this idea, Lastoczi and Klausberger recorded interneurons of unknown classification in CA1 stratum radiatum and lacunosum-moleculare and found that some coupled with 20-45 Hz gamma and others with 60-100 Hz gamma (Lastoczi and Klausberger, 2014). Recent findings raise the possibility that CA3-activated interneurons could drive slow gamma, while also inhibiting fast gamma-generating circuitry. OLM interneurons in CA1, which are activated by inputs from CA3, were shown to inhibit EC input to CA1 while also disinhibiting input from CA3 (Leao et al., 2012). These findings raise the question of whether 
interneurons that receive entorhinal input could operate in a similar manner, driving fast gamma while also inhibiting slow gamma. Experiments that silence select classes of interneurons and determine effects on slow and fast gamma will shed light on this question and the question of which interneuron classes are critically involved in slow and fast gamma generation.

What controls the switch between slow and fast gamma states? During theta-related behaviors, the fast gamma state occurs more often than the slow gamma state (Colgin et al., 2009; Belluscio et al., 2012). Thus, it is possible that fast gamma is the default state during active exploration. Perhaps there is a region in the brainstem or diencephalon that projects to the hippocampus and causes the network to switch from fast gamma to slow gamma. The supramamillary nucleus (SUM) is a potential candidate to initiate the switch. The SUM projects to the hippocampus and entorhinal cortex (Pan and McNaughton, 2004, for a review), and SUM neurons have been reported to exhibit $\sim 37$ ms interspike intervals (Kocsis and Vertes, 1994), which matches the period of slow gamma. However, the relationship between SUM and the hippocampus during slow gamma remains to be tested.

How do slow and fast gamma relate to different populations of gamma-modulated place cells found in CA1 (Senior et al., 2008)? Slow and fast gamma phase distributions for place cell spikes were reported to be different (Colgin et al., 2009), raising the possibility that the two populations reported by Senior and colleagues (2008) represent slow and fast gamma-modulated cells. In line with this idea, place cells reportedly tend to fire on the trough of fast gamma and the rising phase of slow gamma (Colgin et al., 2009). Additionally, place cells that fire on the rising phase of gamma in Senior et al. (2008) fire more tightly locked to theta phase when gamma is present, whereas gamma trough-preferring cells precess across the range of theta phases when gamma is present. These results at first appear similar to effects of slow and fast gamma on theta phase precession reported by Bieri et al. (2014). However, Bieri et al. showed that place cells tend to fire early in their place field during slow gamma. In contrast, cells in Senior et al. that prefer the gamma rising phase fire mostly in the second half of the place field. Thus, the question of how the two populations of gamma-modulated cells reported by Senior et al. relate to slow and fast gamma-modulated cells remains unanswered.

Are slow and fast gamma limited to the hippocampus or do they represent different processing states throughout the brain? Accumulating evidence points to the latter. Separate slow and fast gamma rhythms have been reported in the olfactory bulb (Kay, 2003). Fast gamma $(65-100 \mathrm{~Hz})$ in the olfactory bulb occurred during active intake of olfactory stimuli and during exploratory behaviors, whereas slow gamma $(35-65 \mathrm{~Hz})$ tended to occur during alert immobility. Separate slow $(45-55 \mathrm{~Hz})$ and fast $(70-85 \mathrm{~Hz})$ gamma rhythms have also been reported in the ventral striatum, where they occur during different components of a spatial decision task (van der Meer and Redish, 2009). Also, a recent study in the visual cortex of macaque monkeys found that fast gamma $(\sim 60-80 \mathrm{~Hz})$ rhythms are involved in bottom-up processing, while slower beta ( 14-18 Hz) rhythms mediate top-down processing (Bastos et al., 2014). The beta frequencies observed in this study were lower than the slow gamma frequencies seen in the above-described 
studies. However, the studies described above involved rodents, not monkeys. It is possible that the beta rhythms seen in monkeys during top-down processing are analogous to slow gamma observed in rodents, with lower frequencies perhaps allowing for longer conduction delays (Kopell et al., 2000) in the larger primate brain (Zheng and Colgin, in press).

\section{Conclusions}

It is imperative to continue testing the two-gamma hypothesis and to provide answers to the many questions that remain. The above-described data support the conclusion that the hippocampus takes in and processes information differently depending on gamma state, similar to how theta and sharp wave-ripples represent distinct processing states in the hippocampal network. If this is the case, then future studies will need to take this into consideration when evaluating and interpreting hippocampal results. 
ACKNOWLEDGEMENTS: I acknowledge Sean G. Trettel for collecting the data shown in Figure 1. Funding was provided by the Esther A. and Joseph Klingenstein Fund, the Alfred P. Sloan Foundation, the Whitehall Foundation, grant NIRP-14-305205 from the Alzheimer's Association, and grant N00014-14-1-0322 from ONR.

\section{FIGURE CAPTIONS}

Figure 1: Theta-modulated fast gamma rhythms in MEC during REM sleep. A color-

coded cross-frequency coherence plot is shown on the left. Note how theta phase (x-axis) modulates the power of fast gamma (y-axis). An example recording of theta-modulated fast gamma rhythms is shown on the right. 


\section{REFERENCES}

1. Ahmed, O.J., and Mehta, M.R. (2012). Running speed alters the frequency of hippocampal gamma oscillations. J Neurosci 32, 7373-7383.

2. $\quad$ Ainge, J.A., van der Meer, M.A., Langston, R.F., and Wood, E.R. (2007). Exploring the role of context-dependent hippocampal activity in spatial alternation behavior. Hippocampus 17, 988-1002.

3. Bastos, A.M., Vezoli, J., Bosman, C.A., Schoffelen, J.M., Oostenveld, R., Dowdall, J.R., De Weerd, P., Kennedy, H., and Fries, P. (2014). Visual areas exert feedforward and feedback influences through distinct frequency channels. Neuron, in press.

4. Belluscio, M.A., Mizuseki, K., Schmidt, R., Kempter, R., and Buzsaki, G. (2012). Crossfrequency phase-phase coupling between theta and gamma oscillations in the hippocampus. J Neurosci 32, 423-435.

5. $\quad$ Bi, G.Q., and Poo, M.M. (1998). Synaptic modifications in cultured hippocampal neurons: dependence on spike timing, synaptic strength, and postsynaptic cell type. J Neurosci $18,464-472$.

6. Bieri, K.W., Bobbitt, K.N., and Colgin, L.L. (2014). Slow and fast gamma rhythms coordinate different spatial coding modes in hippocampal place cells. Neuron 82, 670-681.

7. Bragin, A., Jando, G., Nadasdy, Z., Hetke, J., Wise, K., and Buzsaki, G. (1995). Gamma (40-100 Hz) oscillation in the hippocampus of the behaving rat. J Neurosci 15, 47-60.

8. Brun, V.H., Leutgeb, S., Wu, H.Q., Schwarcz, R., Witter, M.P., Moser, E.I., and Moser, M.B. (2008). Impaired spatial representation in CA1 after lesion of direct input from entorhinal cortex. Neuron 57, 290-302.

9. $\quad$ Brun, V.H., Otnass, M.K., Molden, S., Steffenach, H.A., Witter, M.P., Moser, M.B., and Moser, E.I. (2002). Place cells and place recognition maintained by direct entorhinalhippocampal circuitry. Science 296, 2243-2246.

10. Buonomano, D.V. (1999). Distinct functional types of associative long-term potentiation in neocortical and hippocampal pyramidal neurons. J Neurosci 19, 6748-6754.

11. Buzsaki, G. (1986). Hippocampal sharp waves: their origin and significance. Brain Res 398, 242-252.

12. Buzsaki, G., Leung, L.W., and Vanderwolf, C.H. (1983). Cellular bases of hippocampal EEG in the behaving rat. Brain Res 287, 139-171.

13. Cabral, H.O., Vinck, M., Fouquet, C., Pennartz, C.M., Rondi-Reig, L., and Battaglia, F.P. (2014). Oscillatory dynamics and place field maps reflect hippocampal ensemble processing of sequence and place memory under NMDA receptor control. Neuron 81, 402-415.

14. Carr, M.F., Karlsson, M.P., and Frank, L.M. (2012). Transient slow gamma synchrony underlies hippocampal memory replay. Neuron 75, 700-713.

15. Charpak, S., Pare, D., and Llinas, R. (1995). The entorhinal cortex entrains fast CA1 hippocampal oscillations in the anaesthetized guinea-pig: role of the monosynaptic component of the perforant path. Eur J Neurosci 7, 1548-1557.

16. Chrobak, J.J., and Buzsaki, G. (1994). Selective activation of deep layer (V-VI) retrohippocampal cortical neurons during hippocampal sharp waves in the behaving rat. J Neurosci 14, 6160-6170.

17. Chrobak, J.J., and Buzsaki, G. (1998). Gamma oscillations in the entorhinal cortex of the freely behaving rat. J Neurosci 18, 388-398. 
18. Climer, J.R., DiTullio, R., Newman, E.L., Hasselmo, M.E., and Eden, U.T. (2014). Examination of rhythmicity of extracellularly recorded neurons in the entorhinal cortex. Hippocampus 00, 1-14.

19. Colgin, L.L. (2013). Mechanisms and functions of theta rhythms. Annu Rev Neurosci 36, 295-312.

20. Colgin, L.L. (2014). Theta-gamma coupling in the entorhinal-hippocampal system. Curr Opin Neurobiol 31C, 45-50.

21. Colgin, L.L., Denninger, T., Fyhn, M., Hafting, T., Bonnevie, T., Jensen, O., Moser, M.B., and Moser, E.I. (2009). Frequency of gamma oscillations routes flow of information in the hippocampus. Nature 462, 353-357.

22. Colgin, L.L., Kubota, D., Jia, Y., Rex, C.S., and Lynch, G. (2004). Long-term potentiation is impaired in rat hippocampal slices that produce spontaneous sharp waves. $\mathrm{J}$ Physiol 558, 953-961.

23. Csicsvari, J., Jamieson, B., Wise, K.D., and Buzsaki, G. (2003). Mechanisms of gamma oscillations in the hippocampus of the behaving rat. Neuron 37, 311-322.

24. Douchamps, V., Jeewajee, A., Blundell, P., Burgess, N., and Lever, C. (2013). Evidence for encoding versus retrieval scheduling in the hippocampus by theta phase and acetylcholine. $\mathrm{J}$ Neurosci 33, 8689-8704.

25. Fyhn, M., Molden, S., Witter, M.P., Moser, E.I., and Moser, M.B. (2004). Spatial representation in the entorhinal cortex. Science 305, 1258-1264.

26. Hafting, T., Fyhn, M., Molden, S., Moser, M.B., and Moser, E.I. (2005). Microstructure of a spatial map in the entorhinal cortex. Nature 436, 801-806.

27. Hasselmo, M.E., Bodelon, C., and Wyble, B.P. (2002). A proposed function for hippocampal theta rhythm: separate phases of encoding and retrieval enhance reversal of prior learning. Neural Comput 14, 793-817.

28. Igarashi, K.M., Lu, L., Colgin, L.L., Moser, M.B., and Moser, E.I. (2014). Coordination of entorhinal-hippocampal ensemble activity during associative learning. Nature 510, 143-147.

29. Jeewajee, A., Barry, C., O'Keefe, J., and Burgess, N. (2008). Grid cells and theta as oscillatory interference: electrophysiological data from freely moving rats. Hippocampus 18 , 1175-1185.

30. Jensen, O., and Lisman, J.E. (2005). Hippocampal sequence-encoding driven by a cortical multi-item working memory buffer. Trends Neurosci 28, 67-72.

31. Kay, L.M. (2003). Two species of gamma oscillations in the olfactory bulb: dependence on behavioral state and synaptic interactions. J Integr Neurosci 2, 31-44.

32. Kemere, C., Carr, M.F., Karlsson, M.P., and Frank, L.M. (2013). Rapid and continuous modulation of hippocampal network state during exploration of new places. PLoS One 8, e73114.

33. Kocsis, B., and Vertes, R.P. (1994). Characterization of neurons of the supramammillary nucleus and mammillary body that discharge rhythmically with the hippocampal theta rhythm in the rat. J Neurosci 14, 7040-7052.

34. Kopell, N., Ermentrout, G.B., Whittington, M.A., and Traub, R.D. (2000). Gamma rhythms and beta rhythms have different synchronization properties. Proc Natl Acad Sci U S A 97, 1867-1872.

35. Kunec, S., Hasselmo, M.E., and Kopell, N. (2005). Encoding and retrieval in the CA3 region of the hippocampus: a model of theta-phase separation. J Neurophysiol 94, 70-82. 
36. Larson, J., and Lynch, G. (1986). Induction of synaptic potentiation in hippocampus by patterned stimulation involves two events. Science 232, 985-988.

37. Larson, J., Wong, D., and Lynch, G. (1986). Patterned stimulation at the theta frequency is optimal for the induction of hippocampal long-term potentiation. Brain Res 368, 347-350.

38. Lasztoczi, B., and Klausberger, T. (2014). Layer-specific GABAergic control of distinct gamma oscillations in the CA1 hippocampus. Neuron 81, 1126-1139.

39. Leao, R.N., Mikulovic, S., Leao, K.E., Munguba, H., Gezelius, H., Enjin, A., Patra, K., Eriksson, A., Loew, L.M., Tort, A.B., et al. (2012). OLM interneurons differentially modulate CA3 and entorhinal inputs to hippocampal CA1 neurons. Nat Neurosci 15, 1524-1530.

40. Lever, C., Burton, S., Jeewajee, A., Wills, T.J., Cacucci, F., Burgess, N., and O'Keefe, J. (2010). Environmental novelty elicits a later theta phase of firing in CA1 but not subiculum. Hippocampus 20, 229-234.

41. Manns, J.R., Zilli, E.A., Ong, K.C., Hasselmo, M.E., and Eichenbaum, H. (2007). Hippocampal CA1 spiking during encoding and retrieval: relation to theta phase. Neurobiol Learn Mem 87, 9-20.

42. Montgomery, S.M., and Buzsaki, G. (2007). Gamma oscillations dynamically couple hippocampal CA3 and CA1 regions during memory task performance. Proc Natl Acad Sci U S A 104, 14495-14500.

43. Newman, E.L., Gillet, S.N., Climer, J.R., and Hasselmo, M.E. (2013). Cholinergic blockade reduces theta-gamma phase amplitude coupling and speed modulation of theta frequency consistent with behavioral effects on encoding. J Neurosci 33, 19635-19646.

44. Pan, W.X., and McNaughton, N. (2004). The supramammillary area: its organization, functions and relationship to the hippocampus. Prog Neurobiol 74, 127-166.

45. Penttonen, M., Kamondi, A., Acsady, L., and Buzsaki, G. (1998). Gamma frequency oscillation in the hippocampus of the rat: intracellular analysis in vivo. Eur J Neurosci 10, 718728.

46. Pernia-Andrade, A.J., and Jonas, P. (2014). Theta-gamma-modulated synaptic currents in hippocampal granule cells in vivo define a mechanism for network oscillations. Neuron 81, 140152.

47. Rivas, J., Gaztelu, J.M., and Garcia-Austt, E. (1996). Changes in hippocampal cell discharge patterns and theta rhythm spectral properties as a function of walking velocity in the guinea pig. Exp Brain Res 108, 113-118.

48. Schomburg, E.W., Fernandez-Ruiz, A., Mizuseki, K., Berenyi, A., Anastassiou, C.A., Koch, C., and Buzsaki, G. (2014). Theta Phase Segregation of Input-Specific Gamma Patterns in Entorhinal-Hippocampal Networks. Neuron.

49. Senior, T.J., Huxter, J.R., Allen, K., O'Neill, J., and Csicsvari, J. (2008). Gamma oscillatory firing reveals distinct populations of pyramidal cells in the CA1 region of the hippocampus. J Neurosci. 28, 2274-2286.

50. Shirvalkar, P.R., Rapp, P.R., and Shapiro, M.L. (2010). Bidirectional changes to hippocampal theta-gamma comodulation predict memory for recent spatial episodes. Proc Natl Acad Sci U S A 107, 7054-7059.

51. Siegle, J.H., and Wilson, M.A. (2014). Enhancement of encoding and retrieval functions through theta phase-specific manipulation of hippocampus. Elife 3, e03061.

52. Slawinska, U., and Kasicki, S. (1998). The frequency of rat's hippocampal theta rhythm is related to the speed of locomotion. Brain Res 796, 327-331. 
53. Soltesz, I., and Deschenes, M. (1993). Low- and high-frequency membrane potential oscillations during theta activity in CA1 and CA3 pyramidal neurons of the rat hippocampus under ketamine-xylazine anesthesia. J Neurophysiol 70, 97-116.

54. Takahashi, M., Nishida, H., Redish, A.D., and Lauwereyns, J. (2014). Theta phase shift in spike timing and modulation of gamma oscillation: a dynamic code for spatial alternation during fixation in rat hippocampal area CA1. J Neurophysiol 111, 1601-1614.

55. Tort, A.B., Komorowski, R.W., Manns, J.R., Kopell, N.J., and Eichenbaum, H. (2009). Theta-gamma coupling increases during the learning of item-context associations. Proc Natl Acad Sci U S A 106, 20942-20947.

56. Trimper, J.B., Stefanescu, R.A., and Manns, J.R. (2014). Recognition memory and thetagamma interactions in the hippocampus. Hippocampus 24, 341-353.

57. van der Meer, M.A., and Redish, A.D. (2009). Low and High Gamma Oscillations in Rat Ventral Striatum have Distinct Relationships to Behavior, Reward, and Spiking Activity on a Learned Spatial Decision Task. Front Integr Neurosci 3, 9.

58. Yamamoto, J., Suh, J., Takeuchi, D., and Tonegawa, S. (2014). Successful execution of working memory linked to synchronized high-frequency gamma oscillations. Cell 157, 845-857. 59. Zheng, C., Bieri, K.W., Trettel, S.G., and Colgin, L.L. (2014). The correlation between gamma frequency and running speed differs for slow and fast gamma rhythms in freely behaving rats. Society for Neuroscience Annual Meeting Abstracts 751.717.

59. Zheng, C., and Colgin, L.L. (2014). Beta and gamma rhythms go with the flow. Neuron, in press.

60. Zhu, G., Liu, Y., Wang, Y., Bi, X., and Baudry, M. (in press). Different patterns of electrical activity lead to long-term potentiation by activating different intracellular pathways. J Neurosci. 
Figure 1

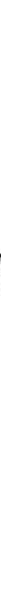

